\title{
ORIGINAL ARTICLE Panel sequencing for clinically oriented variant screening and copy number detection in 142 untreated multiple myeloma patients
}

\author{
KM Kortuem¹, E Braggio ${ }^{1}$, L Bruins ${ }^{1}$, S Barrio ${ }^{1}$, CS Shi ${ }^{1}$, YX Zhu ${ }^{1}$, R Tibes ${ }^{1}$, D Viswanatha ${ }^{2}$, P Votruba ${ }^{3}$, G Ahmann ${ }^{1}$, R Fonseca ${ }^{1}$, \\ P Jedlowski ${ }^{1}$, I Schlam ${ }^{1}$, S Kumar ${ }^{4}$, PL Bergsagel ${ }^{1}$ and AK Stewart ${ }^{1}$
}

We employed a customized Multiple Myeloma (MM)-specific Mutation Panel $\left(M^{3} P\right)$ to screen a homogenous cohort of 142 untreated MM patients for relevant mutations in a selection of disease-specific genes. $M^{3} P v 2.0$ includes 77 genes selected for being either actionable targets, potentially related to drug-response or part of known key pathways in MM biology. We identified mutations in potentially actionable genes in $49 \%$ of patients and provided prognostic evidence of STAT3 mutations. This panel may serve as a practical alternative to more comprehensive sequencing approaches, providing genomic information in a timely and cost-effective manner, thus allowing clinically oriented variant screening in MM.

Blood Cancer Journal (2016) 6, e397; doi:10.1038/bcj.2016.1; published online 26 February 2016

\section{INTRODUCTION}

Multiple Myeloma (MM) is characterized by a remarkably heterogeneous clinical course characterized by early response with recurrent relapse in a majority of patients. To better understand the underlying pathogenesis of the disease, wholeexome or whole-genome sequencing have been performed in $>1000$ MM patients, ${ }^{1-4}$ identifying recurrently mutated genes, as well as dissecting clonal heterogeneity ${ }^{5}$ and clonal evolution. ${ }^{6-8}$ This work has defined the mutation landscape at diagnosis, documented clonal heterogeneity and introduced the concept of therapy selected clonal tides and emergence of drug resistance markers as the genetic hallmarks of the disease. Current genetic diagnostics in MM rely predominantly on fluorescence in situ hybridization and infrequently gene expression profiling but no clinically applicable next-generation sequencing diagnostic is currently available. Our disease-specific MM Mutation Panel ${ }^{9,10}$ in its current version (M3Pv2.0), screens 77 genes relevant to MM (Table 1) and allows both copy number detection using sufficient depth coverage to detect minor subclonal mutations at reduced sample need, cost and processing time. Most importantly, it allows the translation of genomic information from the bench to the bedside, within clinically relevant timeframes.

\section{PATIENTS AND METHODS}

We selected samples of 142 newly diagnosed MM patients from the Mayo Clinic tissue bank. Plasma cells were enriched using anti-CD138+ beads and germline control cells were obtained from peripheral blood mononuclear cells. DNA was extracted using Puregene kit (Qiagen, Hilden, Germany). The average age at diagnosis was 64 years, mean observation time reached 42 months in which 52 death events and 83 progression events occurred. Initial treatment most commonly included combination therapies using lenalidomide/dexamethasone (75/52.8\%), cyclophosphamide/bortezomib/dexamethasone (23/16.2\%), bortezomib/ dexamethasone $(11 / 7.7 \%)$ or bortezomib/lenalidomide/dexamethasone (10/7.04\%). Fluorescence in situ hybridization results were available in all 142 tumor samples, characterizing 52\%/74 hyperdiploid patients, 41\%/58 of del13q and 15\%/21 of high-risk del17p in the selected cohort. Translocations were confirmed in 39\%/55 of cases including a translocation $t(11 ; 14)$ in $26 \% / 37$ patients, $t(4 ; 14)$ in $10 \% / 14$ patients and $t(14,16)$ in $3 \% / 4$ of the patients.

Samples were sequenced using the lonTorrent platform (PGM, Thermo Fisher, Carlsbad, CA, USA) using the $\mathrm{M}^{3} \mathrm{P}$ gene panel. The updated version $\left(M^{3} \mathrm{Pv} 2.0\right)$ extends on its previously published 47 gene version and comprises 77 genes, including (a) genes known to be expressed in MM and being recurrently mutated in $>2 \%$ of cases, (b) genes belonging to disease-relevant pathways (MAPK, NFkappaB, IL6, Cell cycle, MYC), (c) actionable targets (for example, BRAF, IDH1, FGFR3) and (d) genes in pathways targeted by the most commonly used drugs (proteasome inhibitors, immune modulators (IMiDs), corticosteroids). Furthermore, it allows the detection of copy number alterations in the genes incorporated to our panel.

Twenty nanograms of DNA were used to prepare $200 \mathrm{bp}$ libraries (lon AmpliSeq Library Kit 2.0, Thermo Fisher). Template preparation and enrichment of DNA libraries was done on the lon OneTouch2 and lon OneTouch ES (Thermo Fisher) automated system, respectively. Batches of four samples were barcoded (Ion Xpress Barcode Adapters, Thermo Fisher), pooled and sequenced on lon $318 \mathrm{v} 2$ chips using the lon Sequencing 200 Kit v2 (Thermo Fisher). The generated sequencing data were analyzed using the lon Reporter Software v1.6 (Thermo Fisher), then visualized and manually reviewed using the Integrative Genomics Viewer (IGV, Broad Institute, Cambridge, MA, USA). Further genomic annotation was performed using the Biological reference repository ${ }^{11}$ (BioR, Mayo Clinic, Rochester, MN, USA). Somatic variants were considered for analysis, that were either known cancer associated (based on listing in the COSMIC database) or that were called with a minimal coverage of $\times 20$ including $\geqslant 3 \%$ bidirectional variant reads (VR).

\footnotetext{
${ }^{1}$ Division of Hematology Oncology, Mayo Clinic, Scottsdale, AZ, USA; ${ }^{2}$ Division of Medicine and Pathology, Mayo Clinic, Rochester, MN, USA; ${ }^{3}$ Department of Research, Mayo Clinic, Scottsdale, AZ, USA and ${ }^{4}$ Division of Hematology Oncology, Mayo Clinic, Rochester, MN, USA. Correspondence: Dr AK Stewart, Division of Hematology, Mayo Clinic, 13400 East Shea Boulevard, CRB 1-106 Scottsdale, AZ, 85259-5494, USA.
}

E-mail: stewart.keith@mayo.edu

Received 29 September 2015; revised 30 November 2015; accepted 4 December 2015 
Table 1. $\mathrm{M}^{3} \mathrm{Pv} 2.0$ includes 77 genes either known to be recurrently mutated in MM, to belong to disease-relevant pathways (MAPK, NFkappaB, IL6, Cell cycle, MYC), to be potentially actionable or being in pathways targeted by the most commonly used drugs (proteasome inhibitors, immune modulators, corticosteroids)

\begin{tabular}{lllll}
\hline ACTG1 & CSNK2A1 & IKZF3 & PRDM1 & TNFRSF13B \\
ATM & CUL4A & IL6 & PSMA1 & TNFRSF21 \\
B2M & CUL4B & IL6R & PSMB5 & TNFSF9 \\
BAGE2 & CXCR4 & IL6ST & PSMB8 & TP53 \\
BIRC2 & CYLD & IRF4 & PSMB9 & TRAF2 \\
BIRC3 & DIS3 & JAK2 & PSMD1 & TRAF3 \\
BRAF & EGFR & KDM6A & PTPN11 & TRAF3IP1 \\
CARD11 & EGR1 & KRAS & RASA2 & WHSC1 \\
CCNB1 & FAM46C & MAF & RB1 & XBP1 \\
CCND1 & FGFR3 & MAFB & RIPK1 & \\
CCNT1 & GRB2 & MAP3K14 & RIPK4 & \\
CDK4 & IDH1 & MAX & SHC1 & \\
CDK7 & IDH2 & MYC & SP140 & \\
CDKN1B & IDH3A & MYD88 & SRF & \\
CDKN2A & IFNGR2 & NFKBIB & STAT3 & \\
CDKN2C & IGF1R & NR3C1 & TGFBR2 & \\
CRBN & IKZF1 & NRAS & TLR4 & \\
\hline
\end{tabular}

\section{Statistical methods}

Descriptive statistics were used to describe the frequency distribution for the gene mutations of interest. Overall survival was calculated as the time from start of treatment to death due to any cause. Progression-free survival was calculated as the time from start of treatment to the earliest date of progression or death due to any cause. A patient was considered censored at the last follow-up date if the specified event had not been observed. Overall survival (OS) and progression-free survival (PFS) were estimated using the Kaplan-Meier method and compared between patients with and without the gene mutation of interest using a Cox proportional hazards regression model. A hazards ratio (HR) and $95 \%$ confidence interval for each gene mutation of interest was generated. To account for multiple comparisons, we calculated the false discovery rate by the method of Benjamini and Hochberg using the SAS procedure PROC MULTTEST. A false discovery rate of 0.20 was considered acceptable for exploratory purposes. SAS version 9.3 (Cary, NC, USA) was used for analysis.

\section{RESULTS}

We sequenced the tumor samples with an average coverage depth of $\times 658$ and the corresponding germline samples with an average of $\times 492$. We identified 258 somatic variants including 30 InDels (16 frameshift deletions, 9 frameshift insertions and 5 inframe deletions), 22 nonsense, 2 stoploss and 204 missense mutations of which were 10 in splice acceptor and receptor sites. We used Poly-Phen2, SIFT and PROVEAN to estimate the functional impact of each mutation. In total, predictions of 206 mutations were generated and in $179(87 \%)$ of them at least one algorithm predicted a damaging impact of the mutation on the protein structure or the protein function. The mutational spectrum observed was broad, with $>50 \%$ of the variants below $25 \%$ VR and almost a quarter of the mutations (24\%) found in minor subclone with VR $<10 \%$ (Figure 1 ).

Mutation incidence in patients ranged from 0 to 8 (average 1.5), with $75.4 \%$ of the patients and in $47(61 \%)$ of the 77 panel genes having at least one mutation identified. The most commonly mutated genes in the cohort, exceeding $5 \% \mathrm{VR}$, include KRAS (34/24\%), NRAS (24/17\%), DIS3 (20/14\%), TRAF3 (15/11\%), BRAF (13/9\%), TP53 (13/9\%), FAM46C (11/8\%) and CYLD (7/5\%).

Interestingly, in 28 of the patients (15.5\%) we found $\mathrm{M}^{3} \mathrm{P}$ genes harboring multiple mutations in the same gene (range 2-4) affecting 15 of the $77 \mathrm{M}^{3} \mathrm{P}$ genes, which were ACTG, BRAF, CYLD, IRF4, MAX, SP140, TNFRSF21, TP53, TRAF2 (one patient each harboring multiple mutations), ATM, FAM46C (in two patients each), DIS3 (in three patients), CCND1 (in four patients), and KRAS

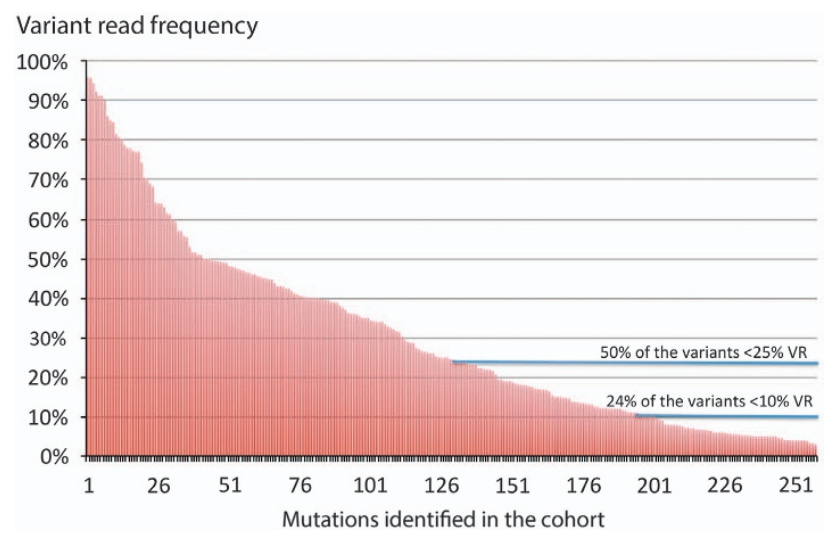

Figure 1. Clonal heterogeneity within the mutations identified, based on the variant read frequency, including a significant proportion of subclonal mutations.

and TRAF3 (in five patients each). Two patients harbored two genes with more than one mutation, with KRAS affected in both cases. (IRF4/KRAS and SP140/KRAS). Of note, the VR frequency in genes with multiple mutations in the same patients, largely included small subclonal mutations with $48 \%$ of them having $<20 \%$ VR.

Mutations affecting the MAPK pathway (NRAS, KRAS, BRAF) were found in $43 \%$ of cases. In total, $92 \%$ of the NRAS/KRAS mutations were identified at known activating hotspots in codon 12, 13 and 61 and known actionable V600E BRAF mutations were present in $46 \%$ of the BRAF mutations. Of note, 10 of the patients with mutations within the MAPK pathway harbored two or more mutations within the pathway. Furthermore, variants were identified in the NFkappaB pathway in $20 \%$ of patients and in the cyclin D1 pathway in $11 \%$ of cases.

Mutations in potentially actionable genes were present in 49\% of our cohort (KRAS, NRAS, BRAF, CCND1, EGFR), according to a recent publication that listed a number of 30 actionable MM genes. ${ }^{2}$ These mutations were most common within the MAPK pathway, additionally we identified a targetable p.Arg132Gly mutation in IDH1 one patient of our cohort. Mutations in the cereblon (CRBN) pathway, known to be essential for responses to IMiD compounds, were present in $6 \%$ of our untreated patient cohort and one patient harbored a mutation in the steroid receptor gene NR3C1 (1\%). Mutations in the IMiD pathway were seen in CRBN, IKZF3, CUL4B (one patient/1\% each) and IRF4 (four patients/3\%, three of which mutated at known hotspot position p.Lys123Arg and one patient harboring four mutations within the IRF4 gene). No mutations in five proteasome subunit genes were observed.

Next, we performed copy number estimation using the lon Reporter v4.0 analysis software (Thermo Fisher) and compared the results with available fluorescence in situ hybridization data for del17p and del13q. We identified good correlation for negative predictive values ( $91 \%$ for del $17 p$ and $80 \%$ for del 13 , respectively) and the specificity (89 and $71 \%$ ) of the test, however, most likely biased by an insufficient amplicon distribution on the affected chromosomes by our panel design, sensitivity (52 and 74\%) and positive predictive value (45 and 64\%) were significantly lower.

Finally, we assessed the impact of each mutated gene (see Supplementary Information and Figure 2) in survival and, even though sample sizes were small, we identified 11 genes in which mutations impacted statistically significant PFS and OS: improved PFS was seen in patients with TRAF3 (hazard ratio 0.32; $95 \%$ confidence interval $(\mathrm{Cl}) 0.12,0.88 ; P$-value $=0.027)$, conversely, shorter PFS was assessed in patients with mutations in STAT3 (HR 3.00; 95\% Cl 1.09, 8.28; $P$-value $=0.034)$, PTPN11 $(\mathrm{HR} 17.01 ; 95 \% \mathrm{Cl}$ 3.73, 77.46; $P$-value < 0.001), PRDM1 (HR 10.22; 95\% Cl 2.31, 45.15; 
$P$-value $=0.002) \quad$ and RIPK1 $(\mathrm{HR} \quad 7.70 ; 95 \%$ Cl 1.02, 58.00; $P$-value $=0.048)$. Shorter OS times was observed by mutation in KRAS (HR 1.97; 95\% Cl 1.08, 3.58; $P$-value $=0.027)$, STAT3 $($ HR 4.92; 95\% Cl 1.91, 12.68, $P$-value $=0.001)$, IRF4 $(\mathrm{HR} 3.39 ; 95 \% \mathrm{Cl}$ 1.03, 11.13; $P$-value =0.044), MAFB (HR 7.85; 95\% Cl 1.03, 59.97; $P$-value $=0.047)$, PTPN11 (HR 8.79; 95\% Cl 2.01, 38.49; $P$-value $=$ $0.004), P R D M 1$ (HR 5.97; 95\% Cl 1.42, 25.09; $P$-value $=0.015)$ and CXCR4 (HR 27.16; 95\% Cl 3.18, 232.30; $P$-value $=0.003$ ).

\section{DISCUSSION}

The mutational landscape of MM is essentially defined with a relatively small number of genes being recurrently mutated in newly diagnosed MM patients. ${ }^{1-4}$ One recent publication that included almost 500 exomes from a homogeneous, untreated cohort, ${ }^{2}$ identified a number of genes that associated with differential survival in MM patients (CCND1, TP53, ATM, ATR, ZFHX4, NCKAP5, IRF, EGR1). We expect, as significant sequencing efforts in $M M$ are ongoing, the number of genes identified to impact survival to specific treatments or in specific MM cohorts will increase. We present in this manuscript the sequencing analysis results of 142 untreated MM tumor samples from patients that underwent variable treatment regimen and we identified different genes with statistically significant impact on survival rates. Owing to the comparably low mutation incidence numbers are small and need validation in larger cohorts. In our cohort STAT3 was the only gene that negatively impacted both PFS and OS when being mutated in our cohort. STAT3-activating mutations have been identified in higher frequencies in various hematologic malignancies with a maximum incidence of $40 \%$ in large granular lymphocytic lymphoma. ${ }^{12}$ However, mutations in this gene have been rarely detected in $\mathrm{MM}$ and in only $3.5 \%$ of our cohort. Overexpression of STAT3 is reported to negatively impact survival in various malignancies, ${ }^{13-15}$ including $\mathrm{MM}^{16}$ and in fact $O S$ and PFS were significantly shorter in our five STAT3-mutated patients (two had other high-risk markers of del17p and $t(4 ; 14)$ by fluorescence in situ hybridization). Three of the five STAT3 variants in our cohort are previously reported including T716M, located in the transactivation domain of the gene associated with the development of early-onset autoimmune diseases $i^{17} \mathrm{Y} 640 \mathrm{H}$, in the $\mathrm{SH} 2$ domain, occurring at the same amino-acid position as a known STAT3-activating mutation $\mathrm{Y} 640 \mathrm{~F} ;{ }^{18-21}$ finally a E616G mutation, known to increase expression of STAT3 in immunohistochemical staining in aggressive B-cell lymphomas ${ }^{18}$ and described as mutated or deleted in multiple B- and T-cell malignancies. $7,15,18,19,22,23$ In addition, in a mouse bone marrow transplantation model, a deletion at same position (del616 STAT3) was shown to induce myeloid malignancy. ${ }^{19}$ Activating mutations in STAT3 are mostly present in the $\mathrm{SH} 2$ domain, but have been also reported in the coiled-coil domain of the gene $\mathrm{e}^{24}$ in which one of the STAT3 mutations from our cohort is located (T277l). Finally, we identified a de novo 5560 C mutation in the DNA-binding domain, for which no further information is available in the literature.

Comparing our data with another recently published untreated cohort, ${ }^{2}$ we observed a slightly increased mutation incidence in our patients, as for example, KRAS ( $24 \%$ vs $21 \%)$, DIS3 ( $14 \%$ vs $9 \%$ ), TRAF3 ( $11 \%$ vs $4 \%)$, BRAF (9\% vs $7 \%)$, FAM46C ( $8 \%$ vs $5 \%)$, or TP53

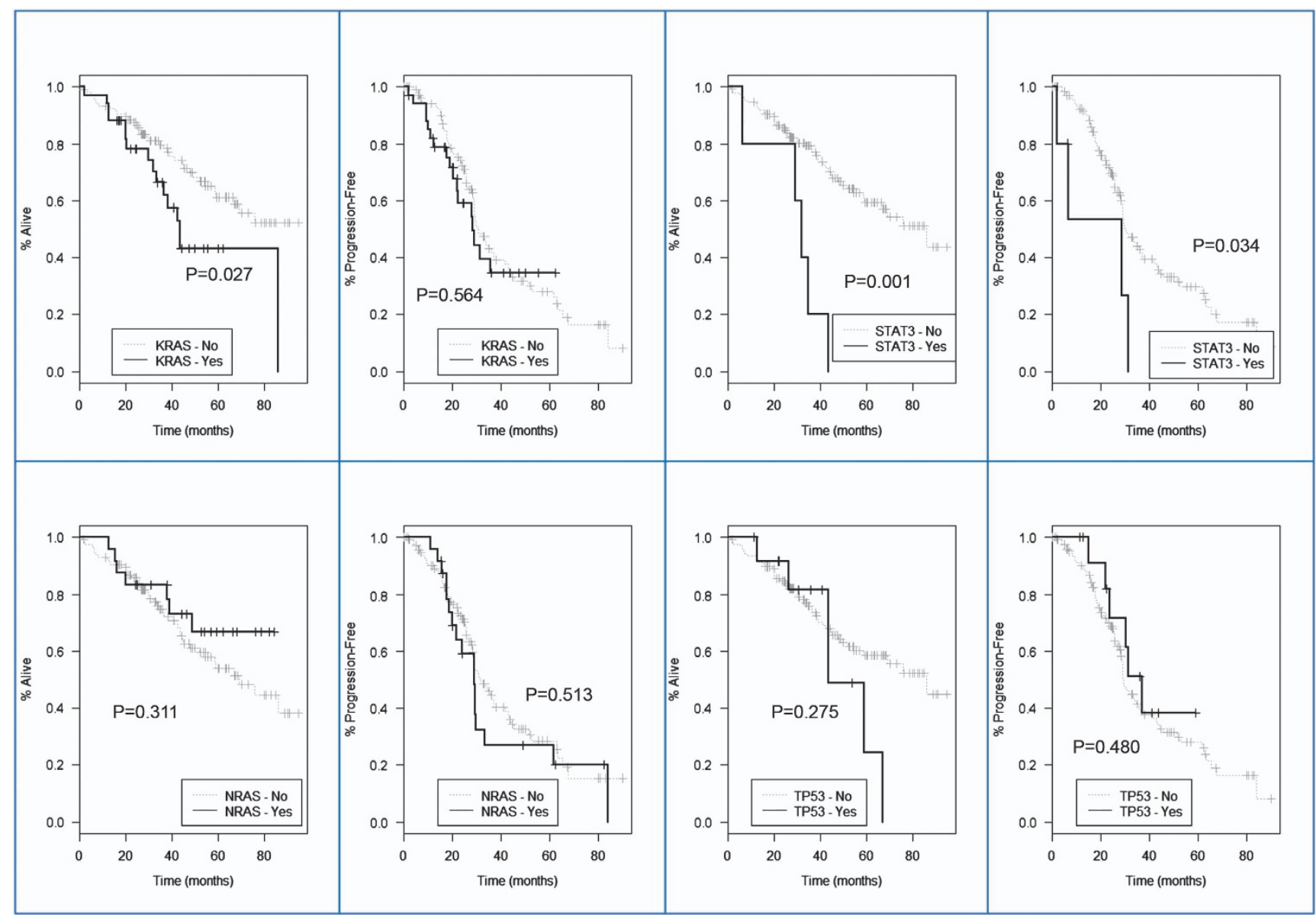

Figure 2. Kaplan-Meier survival estimation for NRAS-, KRAS-, TP53- and STAT3-mutated patients: no statistically significant impact on the survival was observed for NRAS, KRAS or TP53 mutations, but STAT3-mutated patients showed a statistically significantly shortened PFS and OS. 


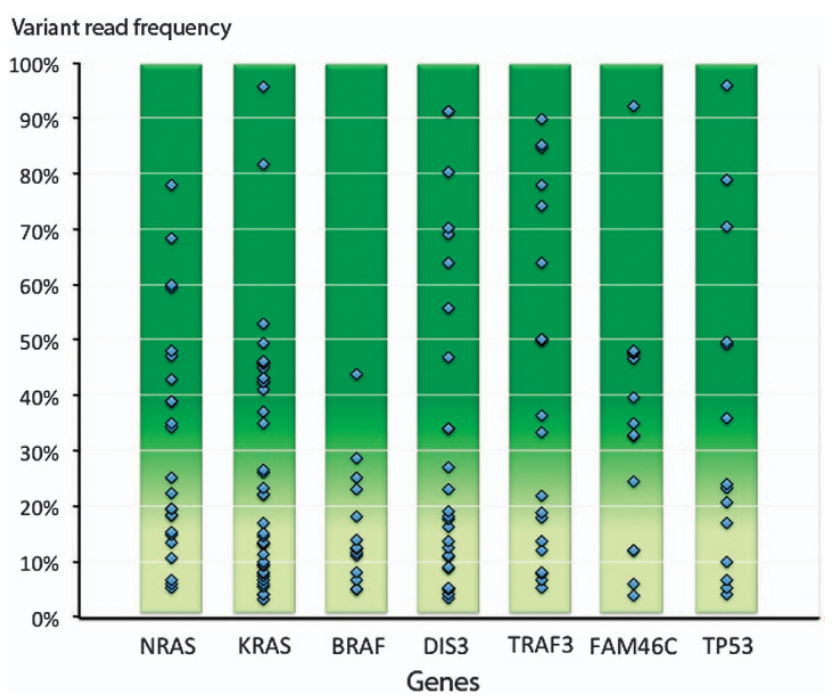

Figure 3. Clonal heterogeneity within most recurrently mutated genes: this includes a significant number of subclonal mutations present in untreated disease, detectable by deep targeted $\mathrm{M}^{3} \mathrm{P}$ sequencing.

( $9 \%$ vs $3 \%$ ). Of note, in these genes we saw a significant number of minor subclonal mutations with VR frequencies $\leqslant 10 \%$, including $31 \%$ of mutations in KRAS, $27 \%$ in DIS3, 20\% in TRAF3, $29 \%$ in $B R A F, 14 \%$ in FAM46C and $29 \%$ in TP53 (Figure 3). Thus, our observation is most likely best explained by the increased sequencing depth of our targeted sequencing approach that allows, compared with usual exome-sequencing parameters, to more sensitively detect minor subclonal mutations. These mutations are of particular interest in longitudinal tumor sample analysis following clonal evolution over time as response to therapy, also being a potential source of later relapse or drug resistance development from such minor subclones has been described.

As expected in an untreated cohort, mutations in genes associated to drug resistance were rare in our cohort with no mutations found in the five $\mathrm{M}^{3} \mathrm{P}$ proteasome subunit coding genes and only one patient harboring a mutation in the steroid receptor $N R 3 C 1$. However, we describe a $6 \%$ mutation incidence of the CRBN pathway (CRBN, CUL4B, IRF4, IKZF1, which might impact response to IMiD treatment. Of interest we identified a $10 \%$ VR mutation in CRBN (p.Asn316Lys) in a patient unresponsive to initial Lenalidomide and later Pomalidomide treatment, thus suggesting an association to the IMiD resistance.

In summary, use of a MM mutation panel might serve as a catalyst to better individualize treatment decisions for the benefit of MM patients. Our targeted sequencing provides a powerful alternative to exome-sequencing approaches and we provide evidence here in support of a MM gene panel, which identified mutations in potentially actionable genes, according to a most recent publication, ${ }^{2}$ in $49 \%$ of newly diagnosed patients, and provided prognostic evidence and guidance with respect to drug resistance and clonal frequency. An updated version of our gene selection $\left(\mathrm{M}^{3} \mathrm{Pv} 3.0\right)$ is currently under clinical validation, in which we incorporated additional targets and an improved amplicon distribution to optimize copy number detection.

\section{CONFLICT OF INTEREST}

The authors declare no conflict of interest.

\section{ACKNOWLEDGEMENTS}

Research funding was obtained from Oncospire genomics. KMK obtained funding from the Deutsche Forschungsgemeinschaft (DFG, KO 4604/1-2; 705 136/807 015). Funding was obtained from Oncospire genomics. KMK obtained funding from the Deutsche Forschungsgemeinschaft (DFG, KO 4604/1-2 (705 136/807 015)).

\section{REFERENCES}

1 Bolli N, Avet-Loiseau H, Wedge DC, Van Loo P, Alexandrov LB, Martincorena I et al. Heterogeneity of genomic evolution and mutational profiles in multiple myeloma. Nat Commun 2014; 5: 2997.

2 Walker BA, Boyle EM, Wardell CP, Murison A, Begum DB, Dahir NM et al. Mutational spectrum, copy number changes, and outcome: results of a sequencing study of patients with newly diagnosed myeloma. J Clin Oncol 2015; 33: 3911-3920.

3 Lonial S, Yellapantula VD, Liang W, Kurdoglu A, Aldrich J, Legendre CM et al. Interim Analysis of the Mmrf Commpass Trial: Identification of Novel Rearrangements Potentially Associated with Disease Initiation and Progression 2014, ASH Meeting abstracts, 06 December 2014, p 722.

4 Lohr JG, Stojanov P, Carter SL, Cruz-Gordillo P, Lawrence MS, Auclair D et al. Widespread genetic heterogeneity in multiple myeloma: implications for targeted therapy. Cancer Cell 2014; 25: 91-101.

5 Brioli A, Melchor L, Cavo M, Morgan GJ. The impact of intra-clonal heterogeneity on the treatment of multiple myeloma. Br J Haematol 2014; 165: 441-454.

6 Melchor L, Brioli A, Wardell CP, Murison A, Potter NE, Kaiser MF et al. Single-cell genetic analysis reveals the composition of initiating clones and phylogenetic patterns of branching and parallel evolution in myeloma. Leukemia 2014; 28: 1705-1715.

7 Keats JJ, Chesi M, Egan JB, Garbitt VM, Palmer SE, Braggio E et al. Clonal competition with alternating dominance in multiple myeloma. Blood 2012; 120: 1067-1076.

8 Egan JB, Shi CX, Tembe W, Christoforides A, Kurdoglu A, Sinari S et al. Wholegenome sequencing of multiple myeloma from diagnosis to plasma cell leukemia reveals genomic initiating events, evolution, and clonal tides. Blood 2012; 120: 1060-1066.

9 Kortum KM, Langer C, Monge J, Bruins L, Egan JB, Zhu YX et al. Targeted sequencing using a 47 gene multiple myeloma mutation panel $(M(3) P)$ in $-17 p$ high risk disease. Br J Haematol 2015; 168: 507-510.

10 Kortum KM, Langer C, Monge J, Bruins L, Zhu YX, Shi CX et al. Longitudinal analysis of 25 sequential sample-pairs using a custom multiple myeloma mutation sequencing panel (M(3)P). Ann Hematol 2015; 94: 1205-1211.

11 Kocher JP, Quest DJ, Duffy P, Meiners MA, Moore RM, Rider D et al. The Biological Reference Repository (BioR): a rapid and flexible system for genomics annotation. Bioinformatics 2014; 30: 1920-1922.

12 Koskela HL, Eldfors S, Ellonen P, van Adrichem AJ, Kuusanmaki H, Andersson El et al. Somatic STAT3 mutations in large granular lymphocytic leukemia. $N$ Engl J Med 2012; 366: 1905-1913.

13 Huang X, Meng B, lqbal J, Ding BB, Perry AM, Cao W et al. Activation of the STAT3 signaling pathway is associated with poor survival in diffuse large B-cell lymphoma treated with R-CHOP. J Clin Oncol 2013; 31: 4520-4528.

$14 \mathrm{Wu}$ ZL, Song YQ, Shi YF, Zhu J. High nuclear expression of STAT3 is associated with unfavorable prognosis in diffuse large B-cell lymphoma. J Hematol Oncol 2011; 4: 31.

15 Qiu ZY, Fan L, Wang L, Qiao C, Wu YJ, Zhou JF et al. STAT3 mutations are frequent in T-cell large granular lymphocytic leukemia with pure red cell aplasia. $J$ Hematol Oncol 2013; 6: 82.

16 Raab MS, Xu J, Hielscher T, Lehners N, Ellert E, Ho AD et al. Signaling Pathway Profiling in Multiple Myeloma 2014, ASH Meeting abstracts, 06 December 2014, p 644.

17 Flanagan SE, Haapaniemi E, Russell MA, Caswell R, Lango Allen H, De Franco E et al. Activating germline mutations in STAT3 cause early-onset multi-organ autoimmune disease. Nat Genet 2014; 46: 812-814.

18 Ohgami RS, Ma L, Monabati A, Zehnder JL, Arber DA. STAT3 mutations are present in aggressive B-cell lymphomas including a subset of diffuse large B-cell lymphomas with CD30 expression. Haematologica 2014; 99: e105-e107.

19 Couronne L, Scourzic L, Pilati C, Della Valle V, Duffourd Y, Solary E et al. STAT3 mutations identified in human hematologic neoplasms induce myeloid malignancies in a mouse bone marrow transplantation model. Haematologica 2013; 98 : 1748-1752.

20 Kucuk C, Jiang B, Hu X, Zhang W, Chan JK, Xiao W et al. Activating mutations of STAT5B and STAT3 in lymphomas derived from gammadelta-T or NK cells. Nat Commun 2015; 6: 6025. 
21 Pilati C, Amessou M, Bihl MP, Balabaud C, Nhieu JT, Paradis V et al. Somatic mutations activating STAT3 in human inflammatory hepatocellular adenomas. $J$ Exp Med 2011; 208: 1359-1366.

22 Lohr JG, Stojanov P, Lawrence MS, Auclair D, Chapuy B, Sougnez C et al. Discovery and prioritization of somatic mutations in diffuse large B-cell lymphoma (DLBCL) by whole-exome sequencing. Proc Natl Acad Sci USA 2012; 109: 3879-3884.

23 Odejide O, Weigert O, Lane AA, Toscano D, Lunning MA, Kopp N et al. A targeted mutational landscape of angioimmunoblastic T-cell lymphoma. Blood 2014; 123: 1293-1296.
24 Hu G, Witzig TE, Gupta M. A novel missense (M206K) STAT3 mutation in diffuse large B cell lymphoma deregulates STAT3 signaling. PloS One 2013; 8: e67851.

\section{(c) (i)}

This work is licensed under a Creative Commons Attribution 4.0 International License. The images or other third party material in this article are included in the article's Creative Commons license, unless indicated otherwise in the credit line; if the material is not included under the Creative Commons license, users will need to obtain permission from the license holder to reproduce the material. To view a copy of this license, visit http://creativecommons.org/licenses/ by/4.0/

Supplementary Information accompanies this paper on Blood Cancer Journal website (http://www.nature.com/bcj) 